MANAGEMENT OF NEXT-GenERATION

WIRELESS NETWORKS AND SERVICES

\title{
Provisioning Multimedia Wireless Networks for Better QoS: RRM Strategies for 3G W-CDMA
}

\author{
Oriol Sallent, Jordi Pérez-Romero, Ramon Agustí, and Ferran Casadevall \\ Universitat Politècnica de Catalunya
}

\begin{abstract}
Third-generation mobile communication systems will bring a wide range of new services with different quality of service requirements and will open the ability to exploit radio resource management functions to guarantee a certain target QoS, to maintain the planned coverage area and to offer a high-capacity while using the radio resources in an efficient way. RRM functions impact the overall system efficiency and the operator infrastructure cost, so they will definitively play an important role in a mature $3 \mathrm{G}$ scenario. In order to provide some insight into RRM strategies implementation, a range of representative case studies with several innovative algorithms arc presented and supported by simulation results in a realistic UMTS Terrestrial Radio Access Network scenario as devised in the 3GGP standardization forum. In particular, a decentralized uplink transmission rate selection algorithm in the short term, a congestion control mechanism to cope with overload situations, and downlink scheduling for layered streaming video packets are proposed.
\end{abstract}

\section{INTRODUCTION}

The evolution of the end user needs towards multimedia applications has pushed the wireless community to conceive the so-called third-generation (3G) systems (e.g., Universal Mobile Telecommunications System, UMTS, or cdma2000), where wideband code-division multiple access (W-CDMA) is the predominant technology. W-CDMA access networks provide an inherent flexibility to handle the provision of future $3 \mathrm{G}$ mobile multimedia services with different quality of service (QoS) guarantees and the ability to optimize the spectrum efficiency in the air interface by means of efficient radio resource management (RRM) algorithms.
The problem faced by a network operator is to offer a system where the number of users is maximized for a given set of QoS requirements. In this problem two aspects can be clearly distinguished: network planning (i.e., the design of the fixed network infrastructure in terms of number of cell sites, cell site location, number and architecture of concentration nodes, etc.) and radio resource allocation (i.e., for a given network deployment, the way radio resources are dynamically managed in order to meet the instantaneous demands of users moving around the network).

In the framework of $2 \mathrm{G}$ time-division multiple access (TDMA)-based mobile systems (e.g., Global System for Mobile Communications, GSM, or IS-54), the main problem is in network planning. The perceived subjective QoS for voice service is mainly controlled through suitable frequency assignment among cell sites in order to provide a sufficient carrier to interference ratio $(\mathrm{C} / \mathrm{I})$. On the other hand, in $2 \mathrm{G}$ the call blocking probability is the other fundamental QoS parameter and is controlled through providing in a first step enough frequencies to a given cell site and in a second step by adding new sites. For a given $2 \mathrm{G}$ network configuration there is an almost constant value for maximum capacity. Additionally, radio resource allocation in the short term (e.g., on the order of tenths/hundreds of milliseconds) has little to do in a scenario where the supported service (e.g., voicc) requires a channel with constant quality and tight delay constraints.

In the framework of $3 \mathrm{G}$ mobile systems the situation is significantly different. First, in WCDMA-based systems there is not a constant value for the maximum available capacity, since it is tightly coupled to the amount of interference in the air interface. Second, the multiservice scenario drops the stringent delay requirement for some services and, consequently, opens the ability to exploit RRM functions to 
guarantee a certain target QoS, to maintain the planned coverage area and offer high capacity while using the radio resources efficiently. It is worth mentioning that RRM functions can be implemented in many different ways, having an expected impact on overall system efficiency and operator infrastructure cost, so RRM strategies will definitively play an important role in a mature $3 \mathrm{G}$ scenario. Additionally, RRM strategies are not subject to standardization, so they can be a differentiation issue among manufacturers and operators.

The problem of addressing the QoS provisioning for wireless multimedia traffic through suitable RRM strategies has gained great momentum during the last years within the framework of $\mathrm{W}$ CDMA schemes. Also, admission control schemes including handoff procedures with different levels of adaptive resource reservation mechanisms [1,2] as well as short-term protocols to schedule transmissions according to their bit error rate (BER) requirements [3] have been widely proposed. Nevertheless, few studics in the open literature [4] regarding these RRM mechanisms approach realistic scenarios aligned with the actual $3 \mathrm{G}$ standards specifications.

In the above context, the objectives of this article are twofold: first, to contribute to the assessment of some QoS concepts as well as to RRM strategies devised accordingly in the framework of a realistic UMTS radio segment scenario as standardized in the 3G Partnership Project (3GPP); and second, to provide some guidelines on how the generic RRM functions can be implemented in the form of specific and innovative algorithms. The algorithms introduced in this article address uplink rate selection, congestion control, and downlink packet scheduling for laycred streaming video. For this purpose, a range of representative case studies carried out in the framework of the IST ARROWS European Collaborative project [5] are presented and supported by simulation results for algorithm evaluation. Thus, the rest of the article is organized as follows. We define the QoS problem in the radio interface. We describe the 3GPP approach for RRM and also how the Radio Resource Control (RRC) protocol is defined to execute RRM algorithm decisions. We propose several new RRM algorithms and accompany them with results obtained by means of a precise UMTS Terrestrial Radio Access frequency-division duplex (UTRA-FDD) system and link-level simulator. Finally, we summarize the main conclusions reached with this work.

\section{QOS AND THE RADIO INTERFACE}

To cope with a certain QoS a bearer service with clcarly defined characteristics and functionalities must be set up from the source to the destination of the service, maybe including not only the UMTS Terrestrial Radio Access Network (UTRAN, plus core network) but also externaI networks [6]. Within the UMTS bearer service, the role of the radio bearer service is to cover all aspects of the radio interface transport over the UTRAN; consequently, RRM strategies will be responsible for assuring the defined QoS in this segment.
The radio interface of the UTRAN is laycred into three protocol layers: the physical layer (L1), the data link layer (L2), and the network layer (L3). Additionally, L2 is split into two sublayers, radio link control (RLC) and medium access control (MAC). On the other hand, the $\mathrm{RLC}$ and $\mathrm{L} 3$ protocols are partitioned in two planes, user and control. In the control plane, L3 is partitioned into sublayers where only the lowest sublayer, denoted radio resource control (RRC), terminates in the UTRAN [6].

Connections between RRC and MAC as well as $\mathrm{RRC}$ and $\mathrm{L} 1$ provide local interlayer control services, and allow the RRC to control the configuration of the lower layers. In the MAC layer, logical channels are mapped to transport channels. A transport channel defines the way in which traffic from logical channels is processed and sent to the physical layer. The smallest entity of traffic that can be transmitted through a transport channel is a transport block (TB). Once in a certain period of time, called a transmission time interval (TTI), a given number of TBs will be delivered to the physical layer in order to introduce some coding characteristics, interleaving, and rate matching to the radio frame. The set of specific attributes are referred as the transport format (TF) of the considered transport channel. Note that the number of TBs transmitted in a TTI indicates that different bit rates are associated with different TFs. As the user equipment (UE) may have more than one transport channel simultaneously, the TF combination (TFC) refers to the sclected combination of TFs. The list of allowed TFCs to be used is referred to as the transport format combination set (TFCS) [6].

\section{THE RRM FRAMEWORK IN UTRA}

RRM strategies have to be applied to both uplink and downlink in a consistent way. In the uplink direction, centralized solutions (i.e., RRM algorithms located at the radio network controller, RNC) may provide better performance compared to a distributed solution (i.e., RRM algorithms located at the UE) because much more $R R M$ relevant information related to all users involved in the process may be available at the RNC. On the contrary, exccuting decisions made by centralized RRM algorithms would be much more costly in terms of control signaling because in this case the UE must be informed about how to operate. Consequently, strategies face the performance/complexity trade-off that usually finds a good solution in an intermediate state where both centralized and decentralized components are present. The 3GPP approach for the uplink could be included in this category, since it can be divided into two parts.

Centralized component (located at the RNC):

- Admission control. It is employed to decidc whether to accept or reject a new connection depending on the interference (or load) it adds to existing connections. With acceptance, a TFCS is decided so that the maximum allowed bit rate is determined.

- Congestion control. It faces situations in which the system has reached a congestion status and therefore the QoS guarantecs
To cope with a

certain QOS a

bearer service

with clearly

defined charac-

teristics and

functionalities

must be set up

from the source

to the

destination of the

service, 'maybe

including not only

the UMTS

network (UTRAN

plus Core

Network) but also

external

networks. 


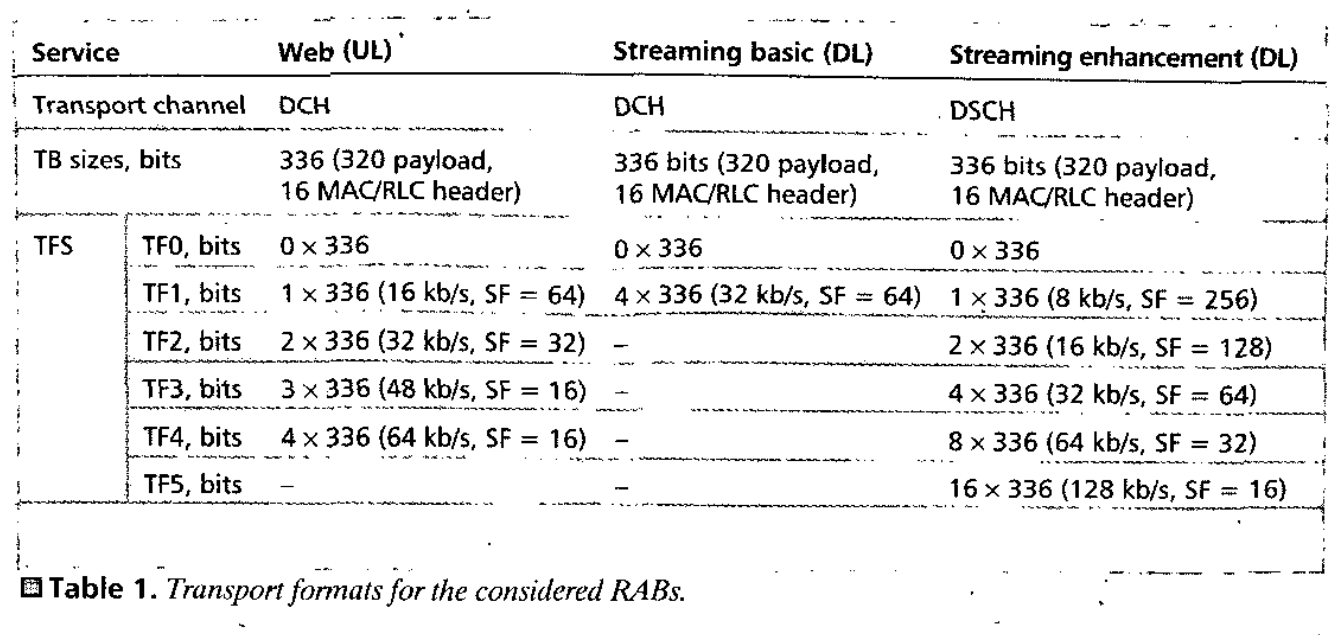

\begin{tabular}{lll} 
UE-MAC strategy & Average packet delay (s) & Rate per page jitter (kb/s) \\
\hline $\mathrm{SCr} 24$ & 0.54 & 5.0 \\
$\mathrm{MR}$ & 0.12 & 11.3 \\
\hline
\end{tabular}

$\square$ Table 2. Average delay performance for UE-MAC algorithms.

are at risk due to the evolution of system dynamics (mobility aspects, increase in interference, etc.). An appropriate selection of TFCS can be seen as a congestion control procedure (limiting the maximum bit rate for example when there is an increase in the measured interfcrence).

Decentralized part (located at UE-MAC): This algorithm autonomously decides a TF for each TTI, and thus operates on a "short" term in order to take full advantage of the time varying conditions. In the uplink, the TF is selected by the MAC layer of each terminal within the TFCS assigned by the network. In this sense, the scheduling is carried out autonomously at the user level, allowing significant signaling savings.

In the downlink, a totally centralized operation arises naturally, and the TF selection can be performed by considering the information of all the users. An additional function, code management, must also be considered. Code management is devoted to manage the orthogonal variable spreading factor (OVSF) code tree used to allocate physical channel orthogonality among different users.

Moreover, handover procedures have a strong impact on the overall RRM, so it is mandatory to develop RRM strategies that take this influence into account. Handover management is in charge of allowing the continuity of the call in progress when the mobile moves from one cell to another and still guaranteeing its QoS.

Decisions made by RRM algorithms are executed through radio bearer control procedures (a subset of RRC procedures) such as [6]:

Radio bearer setup. This procedure is used to set up a new radio bearer and specify a suitable TFCS after performing admission control.

Physical channel reconfiguration. The physical channel reconfiguration in UL can be used to change the transmission frequency band (hard handover), maximum allowed transmission power, minimum W-CDMA spreading factor, and so on. In the downlink, this procedure can also be used to dynamically change downlink OVSF codes allocated to different bearers, taking into account bit rate requirements of each connection.

Transport channel reconfiguration. This procedure is used to change the different parameters of a certain transport channel. Particularly, it can be used to limit the allowed TFCS depending on the system status (e.g., when congestion arises).

\section{RRM ALGORITHM CASE STUDIES}

In this section several case studies involving different RRM strategies are discussed in order to provide some insight on how these algorithms and strategies can be implemented in a real system. The algorithms are evaluated and supported by simulation results, and some representative services are included. In particular, the considered services and their corresponding transmission characteristics are:

Web browsing (interactive class). The radio access bearer considered has a maximum bit rate of $64 \mathrm{~kb} / \mathrm{s}$ in the uplink and an associated $3.4 \mathrm{~kb} / \mathrm{s}$ signaling radio bearer (Table 1 ). The interactive traffic model considers the generation of activity periods (i.e., pages for Web browsing), where several information packets are generated, and a certain thinking time between them, reflecting service interactivity. The specific parameters are: average thinking time bctween pages: $30 \mathrm{~s}$, average number of packet arrivals per page: 25 , number of bytes per packet: average 366 bytes, maximum 6000 bytes (truncated Pareto distribution), time betwecn packet arrivals: average 0.125 s, exponential distribution.

Layered downlink streaming video (streaming class). This service has two different quality layers: basic and enhancement. The radio access bearer considered for the basic layer uses a dedicated channel (DCH) and has two possible TFs: TF0 (no transmission) or TF1 (allowing the transmission of four transport blocks). For the enhancement layer the radio access bearer uses a downlink shared channel (DSCH) and contains 6 TFs, defined in Table 1 , which are selected depending on how the scheduling algorithm behaves. 
The simulation model includes seven cells with radii $0.5 \mathrm{~km}$. Physical layer performance is obtained from link-level simulations carried out to feed the system level simulator presented here with block error rate (BLER) statistics. The mobility model and propagation models are defined in [6].

\section{CASE Study 1: Uplink UE-MAC Algorithm}

This algorithm autonomously decides the transmission rate on a frame-by-frame basis, and thus operates on a "short" term in order to take full advantage of the time-varying conditions. This decision is taken on a decentralized way by the MAC layer of each UE among the possibilitics contained within TFCS. Notice that the inclusion of no transmission is allowed as a particular case of TF. In this case two approaches are explored for interactive-like services (e.g., Web browsing).

Service Credit ( $\mathrm{SCr}$ ) algorithm: This algorithm aims to offer a negotiated average bit rate. The $\mathrm{SCr}$ of a connection accounts for the difference between the obtained bit rate and the expected bit rate for this connection. Essentially, if $\mathrm{SCr}<0$, the connection has obtained a higher bit rate than expected, and if $\mathrm{SCr}>0$, the connection has obtained a lower bit rate than expected. In cach TTI, the SCr for a connection should be updated as follows:

$$
\begin{aligned}
\operatorname{SCr}(n) & =\operatorname{SCr}(n-1) \\
& +(\text { Guaranteed_rate/TB_size }) \\
& - \text { Transmitted_TB }(n-1)
\end{aligned}
$$

where $\operatorname{SCr}(n)$, measured in number of transport blocks per TTI, is the service credit for TTI $=n$, $\mathrm{SCr}(n-1)$ is the service credit in the previous TTI, Guaranteed_rate is the number of bits per TTI that would be transmitted at the guaranteed bit rate, TB_size is the number of bits of the TB for the considered RAB, and Transmitted $\mathrm{TB}(n$ -1 ) is the number of successfully transmitted TB in the previous TTI. At the beginning of the connection: $\operatorname{SCr}(0)=0$.

Maximum Rate (MR) algorithm: This algorithm selects the TF that allows the highest transmission bit rate according to the amount of bits in the buffer waiting for transmission,

Results.- One important measurement to understand the behavior of the different UEMAC strategics is the transport format distribution used. Referring to Table 1, UE-MAC has the freedom to choose among TF0 (when the buffer is empty or $\mathrm{SCr}<0$ ), TF $1, \mathrm{TF} 2, \mathrm{TF} 3$, and TF4. For SCr24 (Fig. 1, SCr24 stands for a service credit strategy with a guaranteed rate of $24 \mathrm{~kb} / \mathrm{s}$ ) it can be observed that most of the time TF 1 and TF2 are used because the UE buffer queues several packets and tends to transmit the information at $24 \mathrm{~kb} / \mathrm{s}$. In turns, in the periods when the UE buffer is empty the UE gains service credits, and when a new packet arrives the transmission rate is increased over the guaranteed one (i.e., TF3 and TF4 are used). For the MR strategy, since it chooses the TF according to the buffer occupancy and tries to transmit the information as fast as possible, most of the time TF4 is used (Fig. 2). Additionally, Table 2 shows the average delay performance for both MR and SCr strategies. It can

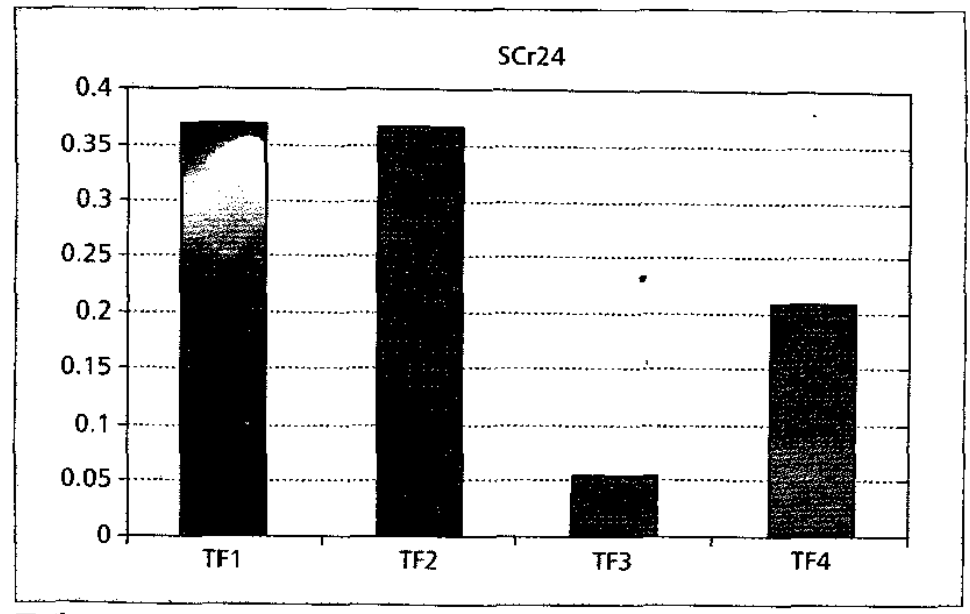

Figure 1.TF distribution for $S C r$.

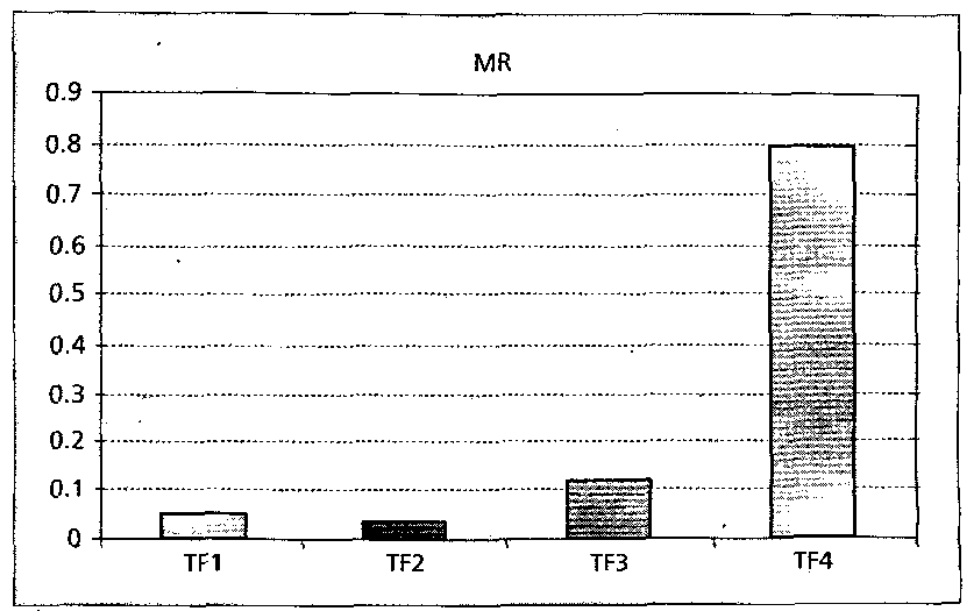

Figure 2. TF distribution for $M R$.

be seen that MR provides lower delay because it tends to maximize the transmission rate according to buffer occupancy. On the contrary, since $\mathrm{SCr}$ does not take buffer occupancy into account, it provides better control of the transmission rate, reflected in a low rate per page jitter. Notice that for the same user and service, depending on the spccific UE-MAC algorithm used, the load or equivalently, the interference this user will cause the system will be different, which should be taken into account in the admission control phase. To raise this effect, consider common uplink admission control based on statistical cell load control, so a call request is only accepted if the resulting cell load $\eta$ is below a certain threshold $\eta_{\max }$ obtained from radio network planning [7]:

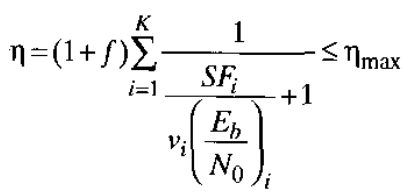

where $S F_{i}$ is the $i$ th user spreading factor, $\left(E_{b} / N_{0}\right)_{i}$ stands for the $i$ th user requirement, $v_{i}$ is the $i$ th user activity factor, $K$ the number of 


\begin{tabular}{|c|c|c|c|c|c|}
\hline \multirow[b]{2}{*}{$\begin{array}{l}\text { Number of } \\
\text { Web users }\end{array}$} & \multicolumn{2}{|c|}{ Algorithm 1 (TFO) } & \multicolumn{3}{|c|}{ Algorithm 2 (TF2) } \\
\hline & $\begin{array}{l}\text { Admission } \\
\text { probability }\end{array}$ & $\begin{array}{l}\text { Time in } \\
\text { congestion (\%) }\end{array}$ & $\begin{array}{l}\text { Admission } \\
\text { probability }\end{array}$ & $\begin{array}{l}\text { Time in } \\
\text { congestion }(\%)\end{array}$ & , \\
\hline 600 & 1 & $\approx 0$ & $1 \ldots \ldots$ & $\approx 0$ & \\
\hline 650 & 1 & $\Rightarrow 0$ & 1 & $\approx 0$ & \\
\hline 700 & 1 & 0.13 & 0.99 & 0.57 & \\
\hline
\end{tabular}

abale 3. Results for $\eta_{\mathrm{CD}}=0.8, \eta_{\mathrm{CR}}=0.7, \Delta \mathrm{T}_{\mathrm{CD}}=10, \Delta \mathrm{T}_{\mathrm{CR}}=10$.

\begin{tabular}{|lcc|}
\hline $\begin{array}{l}\text { Packet delay percentiles } \\
\text { during congestion periods }\end{array}$ & Algorithm 1 & Algorithm 2 \\
\hline $50 \%$ & $<0.12 \mathrm{~s}$ & $<0.16 \mathrm{~s}$ \\
\hline $75 \%$ & $<0.84 \mathrm{~s}$ & $<1.12 \mathrm{~s}$ \\
\hline $95 \%$ & $<2.94 \mathrm{~s}$ & $<6.62 \mathrm{~s}$ \\
\hline
\end{tabular}

Table 4. Results for $\eta_{\mathrm{CD}}=0.8, \eta_{\mathrm{CR}}=0.7, \Delta \mathrm{T}_{\mathrm{CD}}=10, \Delta \mathrm{T}_{\mathrm{CR}}=10$ and 700 users.

users in the cell, and $f$ the intercell to intracell interference factor estimation.

It can be seen from Eq. 1 that for load estimation purposes a certain $S F_{i}$ needs to be considered. Consequently, it can be concluded that the UE-MAC algorithm impacts the admission control process, which should take this fact into account for accurate cell load estimation. For further details on other UE-MAC algorithms and statistical performance measurements see [5].

Case Study 2: Uplink Congestion Control A congestion control mechanism including the following parts is presented for interactive services:

1) Congestion detection: The criterion introduced in order to decide when the network is in congestion and trigger the congestion resolution algorithm is when the load factor increases over a certain threshold, $\eta_{C D}$, during a certain amount of time, $\Delta T_{C D}$ (i.e., if $\eta \geq \eta_{C D}$ in, e.g., 90 percent of the frames within $\left.\Delta T_{C D}\right)$.

2) Congestion resolution: When congestion is assumed in the network, some actions must be taken in order to maintain network stability. The congestion resolution algorithm executes a set of rules to lead the system out of congestion status. Three steps are identified:

a. Prioritization: Ordering the different users from lower to higher priority (i.e., from those that expect a lower grade of service to those with more stringent QoS requirements) in a prioritization table.

b. Load reduction: Two main actions can be taken:

- No new connections are accepted while in congestion.

- Reduce the TFCS (i.e., limit the maximum transmission rate) for a certain number of users already accepted in the network, beginning from the top of the prioritization table.

Algorithm 1: The user is not allowed to transmit anymore while in a congestion period (e.g., the TFCS is limited to TFO by sending from the RNC to the UE the L3 RRC protocol message Transport Channel. Reconfiguration).

Algorithm 2: The TFCS is limited to TF2, so users are not allowed to transmit at more than $32 \mathrm{~kb} / \mathrm{s}$, whereas in normal conditions the maximum rate is $64 \mathrm{~kb} / \mathrm{s}$.

c. Load check: After the actions taken in b), one would recheck the conditions that triggered the congestion status. If congestion persists, one would go back to $b$ ) for the following group of users in the prioritization table. It could be considered that the overload situation has been overcome if, for a certain amount of time $\Delta T_{C R}$ the load factor is below a given threshold, $\eta_{C R}$ (i.e., if $\eta \leq \eta_{C R}$ in,e.g., 90 percent of the frames within $\Delta T_{C R}$ ).

3) Congestion recovery: A congestion recovery algorithm is needed in order to restore to the different mobiles the transmission capabilities they had before the congestion was triggered. Jt is worth mentioning that such an algorithm is crucial because depending on how the recovery is carried out the system could fall again in congestion. A "time scheduling" algorithm (user by user restoring approach) is considered. That is, a specific user is again allowed to transmit at a maximum rate (i.e., a Transport Channel Reconfiguration message indicating that TFCS includes up to TF4 is sent). Once this user has emptied the buffer, another user is allowed to recover the maximum rate, and so on.

Results - Comparisons for the two presented load reduction algorithms are summarized in Tables 3 and 4 . The performance figures are the admission probability (i.e., the probability that a user request is accepted into the system), the percentage of time the network is congested, and the delay distribution of the transmitted packets during the congestion period. It can be observed that the "softer" load reduction actions taken by algorithm 2 lead to more tjme in congestion and consequently a reduction in admission probability (notice that the first action in congestion is to reject all connection requests). It seems that the firmer actions taken by algorithm 1 result in shorter congestion periods. Additionally, one of the expected impacts of congestion is a delay degradation due to the transmission rate capabilities limitation. It can be observed in Table 4 that algorithm 1 provides a nicer delay distribution than algorithm 2 , specially for the 95 th percentile.

\section{CASE Study 3: \\ DOWNLINK PACKET SCHEDULING FOR LAYERED STREAMING VIDEO}

In this case study we focus on streaming video service, which is one of the expected interests in $3 \mathrm{G}$ systems. Quality requirements deal with the achieved bit rate, percentage of lost packets, and delay jitter (rather than the end-to-end delay). It is considered that streaming service allows an initial setup delay that gives room for some 
packet transmissions before the video is reproduced. These packets can be stored in the mobile terminal buffer. Then, with proper buffer dimensioning, the user can be unaware of possible packet retransmissions because the stored buffer allows for continuous packet flow reproduction. Thus, this property gives some more room for scheduling the streaming service since packet retransmissions may play a role.

In order to differentiate quality levels, we assume for this service a two-layered video application that is characterized by two different flows: a basic layer, with the minimum requirements for a suitable visualization, and an enhancement layer that contains additional information to improve the quality of the received images. We will assume that the basic layer will be transmitted through the DCH, while the enhancement layer will be transmitted only if there is capacity.in the DSCHs. It is assumed that the possible retransmissions of the basic layer can be carried out in the $\mathrm{DSCH}$ together with the cnhancement laycr, and having higher priority than the latter.

The proposed packet scheduling strategy allocates resources to the different flows that make use of the DSCH channel. It operates on a frame-by-frame basis (i.e., every $10 \mathrm{~ms}$ ) as follows:

Prioritization: The first step consists of ordering the different users' requests in the DSCH depending on the following priority criteria:

- The higher the number of basic layer TBs to be retransmitted, the higher the priority.

- For the same number of basic layer TBs, the priority is established according to the service credit concept, as explained earlier. The higher the service credit of the enhancement layer. the higher the priority.

Resource allocation: Once requests are ordered, the next step consists of deciding whether or not they are accepted for transmission in the DSCH channel, and which is the accepted TF. A transmission is granted only if the estimated load factor is below a certain threshold $\phi$, and the estimated transmitted power level is below a fraction $\delta$ of the maximum transmitted power. Otherwise, the TF is reduced by one, or equivalently, the transmission bit rate is reduced and the conditions rechecked. If this is not possible, the request should wait for the next frame.

Results - One of the most relevant parameters in the design of the packet scheduling algorithm relies on the threshold $\phi$. Figure 3 presents the average bit rate obtained during a streaming session for the enhancement layer for different $\phi$ values and number of users in the system. $\delta=1$ has been assumed. The basic layer is not presented since its achieved bit rate is almost always $32 \mathrm{~kb} / \mathrm{s}$ (i.e., the basic layer is guaranteed). It can be concluded that the selection $\phi=0.95$ provides the best behavior, since the enhancement layer gets the highest possible bit rate for the different load leveIs. In turns, Fig. 4 plots the delay jitter achieved for different $\phi$ and 120 users. Again $\phi=0.95$ is revealed to be a suitable value because it provides the lowest possible delay variation.

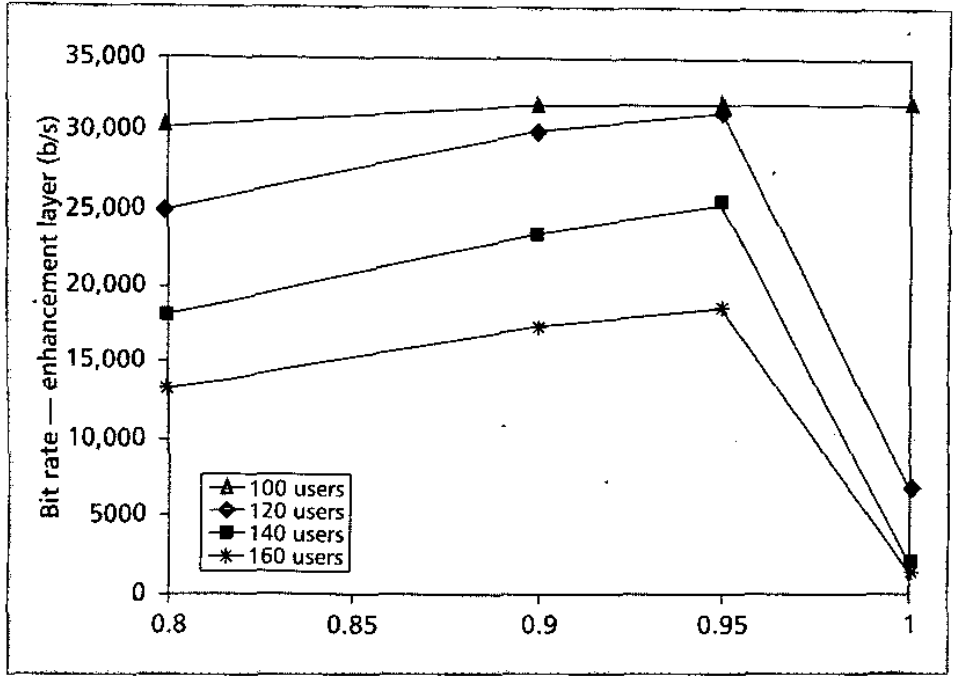

Figure 3. Achieved bit rate.

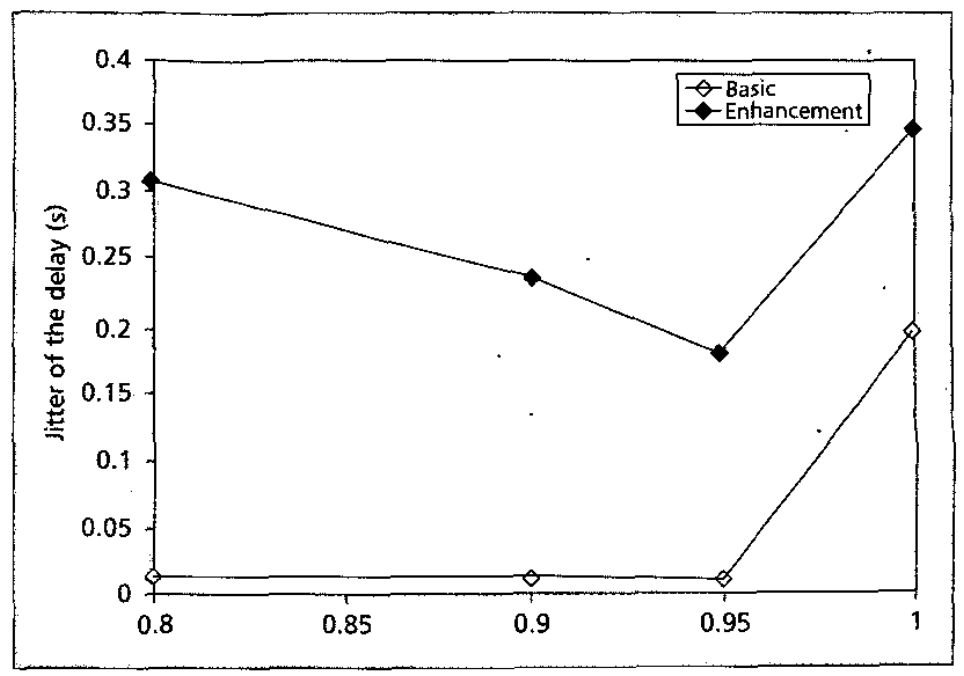

Figure 4. Delay jitter.

\section{CONCLUSIONS}

In this article the key role RRM strategies will play in a mature $3 \mathrm{G}$ W-CDMA based system such as UTRA-FDD has been strengthened. On one hand, $3 \mathrm{G}$ will bring a wide range of new services with different QoS requirements and will open the ability to exploit RRM functions. On the other hand, W-CDMA performance is tightly coupled to the amount of interference in the air interface and eventually depends on many strongly interrelated system parameters that need to be suitably managed through RRM strategies in order to achieve high efficiency.

This article has also proposed several specific RRM algorithms in order to provide some insight to the reader on how the generic RRM functions can be implemented. In particular, the presented case studies have led us to conclude the following:

- Results for UE-MAC strategies show that $\mathrm{SCr}$ tends to use a more stabilized dynamic transmission rate around the agreed value, while 


W-CDMA
performance is
tightly coupled to
the amount of
interference in
the air interface
and eventually
depends on
many strongly
interrelated system
parameters that
need to be
suitably managed
through RRM
strategies in order
to achieve a high
efficiency.

for MR most of the time the highest possible transmission rate is used. This different behavior of the UE-MAC algorithms means that, for the same user and service, depending on the specific UE-MAC algorithm used the load or, equivalently, interference this user will cause the system will be different. Thus, the UE-MAC algorithm impacts on the admission control process, which should take this fact into account for accurate cell load estimation.

-When managing congestion situations, it seems more suitable to adopt strict actions, such as not allowing delay-tolerant services to transmit during the congestion, than softer policies, such as reducing to some extent the transmission rate. With the strict policy the time the network is in congestion is reduced and the delay degradation is nicer than in the softer case.

- Layered streaming video download allows for retransmissions and variable quality. By supporting this service through a dedicated channel for the basic layer and a shared channel for the enhancement layer and retransmissions, high efficiency can be achieved with a proper scheduling algorithm, and OVSF code is saved with the use of the DSCH.

\section{ACKNOWLEDGMENTS}

This work is part of the ARROWS project, partially funded by the European Commission under the IST framework (IST 2000-25133) and the Spanish Research Council under grant TIC20002813-CE. The authors wish to express their gratitude to the other members of the consortium (Telecom Italia Labs, Telefónica I+D, University of Limerick, and Inesc Porto) for valuable discussions, with special thanks to Claudio Guerrini and Paolo Goria from TILab. The help provided by $\mathbf{J}$. Sanchez on some of the simulation work is also acknowledged.

\section{REFERENCES}

[1] S. K. Das et al. "A Call Admission and Control Scheme for QoS Provisioning in Next Generation Wireless Networks," Wireless Networks 6, 2000, pp. 17-30.

[2] Z. Lui and M. El Zarki, "SIR Based Call Admission Control for DS-CDMA Cellular Systems," IEEE JSAC, vol. 12 1994.

[3] I. F. Akyldiz, D. A. Levine, and I. Joe, "A Slotted CDMA Protocol with BER Scheduling for Wireless Multimedia Networks," IEEE/ACM Trans. Net., vol. 7, no. 2, Apr 1999, pp. $146-58$

[4] W. Rave et al., "Evaluation of Load Control Strategies in an UTRAFDD Network," Proc. VTC '01, pp. 2710-14.

[5] http://www.ist-arrows.upc.es

[6] http://www.3gpp.org

[7] H. Holma and A. Toskala, Eds., W-CDMA for UMTS, Wiley, 2000.

\section{BIOGRAPHIES}

ORIOL SALLENT [M'98] (oriol@tsc.upc.es) received Engineer and Doctor Engineer degrees in telecommunication from the Universitat Politèenica de Catalunya (UPC), Spain, in 1994 and 1997, respectively. He joined the Escola Tècnica Superior d'Enginyeria de Telecomunicació de Barcelona, where he became assistant professor in 1994 and associate professor in 1998. His research interests are in the field of mobile communication systems, especially packet radio techniques and radio resource management for spreadspectrum systems. He received the Doctorate Award from the Telecommunication Engineer Association of Spain in 1997 for his Ph.D. dissertation on multiple access protocols for CDMA-based systems.

JORDI PÉREZ-ROMERo [S'98] (jorperez@tsc.upc.es) obtained an Engineer degree in telecommunications from the Escola Tècnica Superior d'Enginyeria 'de Telecomunicació of UPC in 1997. He joined the Radio Communications Group in the Department of Signal Theory and Communications at UPC where he obtained a Ph.D. degree in April 2001. He is currently assistant professor in the field of radio communications. He has been involved in different European projects, and his main research areas are the packet transmission mechanisms and the radio resource management strategies for CDMA mobile communications networks.

FERnANDo J. CASADEVALL [M'87] (ferranc@tsc.upc.es) received Engineer of Telecommunication and Dr.Eng. degrees from UPC in 1977 and 1983, respectively. In 1978 he joined UPC, where he was an associate professor from 1983 to 1991. He is currently a full professor in the Signal Theory and Communications Department. After graduation he was concerned with equalization techniques for digital fibe optic systems. He has also worked in the field of digital communications with particular emphasis on digital radio and its performance under multipath propagation conditions. In the last 10 years, he has mainly been concerned with the performance analysis and development of digital mobile radio systems. In particular, his research interests include cellular and personal communication systems, multipath transceiver design (including software radio techniques), mobility, and radio resource management. Over the last 10 years he participated in more than 20 research projects funded by both public and private organizations. In particular, he participated in the research project CODIT and ATDMA in RACEII and RAINBOW in. ACTS, both funded by the European Commission. Currently in the context of the 5th European Framework Program he participates in the IST projects WINEGLAS and ARROWS, as project manager of the latter. He has published more than 50 technical papers in international conferences and magazines. From October 1992 to January 1996 was responsible for the Information Technology Area in the National Agency for Evaluation and Forecasting (Spanish National Research Council).

RAMON AGUSTI [M'78] (ramon@tsc.upc.ès) received an Engineer of Telecommunications degree from the Universidad Politécnica de Madrid, Spain, in 1973, and a Ph.D. degree from UPC in 1978. In 1973 he joined the Escola Tècnica Superior d'Enginyers de Telecomunicació de Barcelona, Spain, where he became a full professor in 1987. After graduation he worked in the field of digital communications with particular emphasis on transmission and devel opment aspects in fixed digital radio, both radio relay and mobile communications. In this framework he spent six months at the Polithecnic of Turin, Italy, 1976-1977, and one month per year at the CNET laboratories, Lannion, France, from 1980 to 1984 . For the last 15 years he has mainly been concerned with the performance analysis and development of planning tools and equipment for mobile communication systems, and he has published about 100 papers in these areas. He participated in the European program COST 231 (1989-1996), Evolution of Land Mobile Radio, and in COST 259 (Wireless Flexible Personalized Communications) as Spanish delegate. He also participated in RACE and ACTS European research programs in the past (ATDMA, MICC, and RAINBOW projects) and in IST at the present (WINE GLASS and ARROWS projects) as well as in many private and public funded projects. In this time he has also been an advisor to Spanish and Catalonian governmental agencies (DGTel, CICYT, ANEP, and CIRIT) on issues concerning mobile communications. He received the Catalonia Engineer of the Year prize in 1998. He is part of the editorial board of several scientific international journals. At present his research interest lies in the area of mobile communications with special emphasis on CDMA systems, packet radio networks, radio resource allocation, and QoS. 